Revue Française de Civilisation Britannique

XVII-2 | 2012

Minorités, intégration en Grande-Bretagne et dans les pays du Commonwealth

\title{
Visibilités sikhe et musulmane au Royaume-Uni : tentative de comparaison
}

Sikh and Muslim Visibilities in the Uk: an Attempted Comparison

\section{Vincent Latour}

\section{CpenEdition}

Journals

Édition électronique

URL : http://journals.openedition.org/rfcb/683

DOI : $10.4000 /$ rfcb.683

ISSN : 2429-4373

Éditeur

CRECIB - Centre de recherche et d'études en civilisation britannique

Édition imprimée

Date de publication : 15 octobre 2012

Pagination : 145-162

ISBN : 2-911580-37-0

ISSN : 0248-9015

Référence électronique

Vincent Latour, "Visibilités sikhe et musulmane au Royaume-Uni : tentative de comparaison », Revue Française de Civilisation Britannique [En ligne], XVII-2 | 2012, mis en ligne le 15 mars 2016, consulté le 04 mai 2019. URL : http://journals.openedition.org/rfcb/683 ; DOI : 10.4000/rfcb.683

Ce document a été généré automatiquement le 4 mai 2019.

\section{c) () $९$}

Revue française de civilisation britannique est mis à disposition selon les termes de la licence Creative Commons Attribution - Pas d'Utilisation Commerciale - Pas de Modification 4.0 International. 


\section{Visibilités sikhe et musulmane au Royaume-Uni : tentative de comparaison}

Sikh and Muslim Visibilities in the Uk: an Attempted Comparison

Vincent Latour

1 Au cours de la décennie passée (émeutes interethniques dans le Nord et les Midlands, attentats du 11 septembre et du 7 juillet 2005), le débat politique, universitaire et médiatique sur le multiculturalisme en tant que mode d'incorporation s'est focalisé de manière quasi exclusive (voire, obsessionnelle) sur la place de l'Islam et des musulmans dans la société britannique. À cet égard, le discours prononcé à Munich par David Cameron le 5 février 2011, si abondamment commenté, illustre l'ampleur de cette fixation, mais également sa permanence depuis les attentats du 11 septembre, voire depuis l'affaire Rushdie. En effet, l'irruption de la «communauté musulmane» sur le devant de la scène politico-médiatique britannique à la fin des années 1980 suite à la publication des Versets Sataniques tend à occulter la mobilisation coordonnée et constante des Sikhs du Royaume-Uni au cours des cinquante dernières années. Ils devancèrent largement les musulmans et les autres groupes minoritaires issus de l'immigration dans la formulation des demandes de reconnaissance culturelle.

Dix ans après les débuts de la remise en cause du «consensus multiculturel » né des émeutes des années 1980, il semble opportun de s'interroger sur deux stratégies distinctes de la visibilité, afin d'en identifier points de divergence et de convergence.

Cet article s'articulera autour de deux grandes parties. La première dressera un historique de leur présence au Royaume-Uni et s'intéressera aux prémices des visibilités musulmane et sikhe outre-Manche, notamment par le biais des aspects cultuels. La deuxième tentera de mettre en parallèle les modalités de structuration communautaire et de demande de reconnaissance publique de ces deux groupes, y compris dans un contexte de remise en cause apparente du multiculturalisme ${ }^{1}$. 


\section{Historique de la présence des deux groupes religieux au Royaume-Uni et premiers pas vers la visibilité}

Les musulmans du Royaume-Uni (environ 1,6 millions d'après le recensement de 2001², mais qui pourraient être 2,4 millions selon des projections de novembre $2008^{3}$, dans l'attente des résultats du recensement de 2011) sont pour quasiment les trois quarts (74\% ) issus du sous-continent indien ${ }^{4}$. L'obédience très majoritaire en leur sein est le sunnisme, au sein duquel deux grands groupes spécifiquement issus du sous-continent indien se détachent. D'une part, les déobandis, ( $\mathrm{du}$ nom de la ville de Déoband, aujourd'hui dans l'état de l'Uttar Pradesh, dans le Nord de l'Inde), qui revendiquent l'héritage d'un grand juriste des premiers siècles de l'islam, l'imam Abou Harifa ( $45 \%$ des lieux de culte). D'autre part, les barelwis, qui se réclament d'un mouvement lancé en 1880 , notamment dans le but de contrer certains courants réformistes $(25 \%$ des mosquées britanniques). Les salafistes ( $6 \%$ ) et mawdudistes (3,3\%) sont, par contraste, très minoritaires. On trouve également diverses écoles de pensée de tradition sunnite très minoritaires, ainsi que des Chiites (4,5\% des mosquées), souvent originaires d'Iran, Irak, du Liban, du Yémen ou du Koweït. ${ }^{5}$ Il est à noter que les ismaéliens, minorité musulmane indo-pakistanaise très bien intégrée du point de vu socio-économique au Royaume Uni ${ }^{6}$, appartiennent à une branche du chiisme.

5 Même si dès le XVIII siècle des marins de confession musulmane originaires du Bengale ou du Gujarat séjournèrent sporadiquement dans les grandes villes portuaires (Liverpool, Bristol, Cardiff ou Londres), la première vague importante d'installation (le plus souvent temporaire) de musulmans remonte à la deuxième partie du règne de Victoria, puisqu'on estime que 70 à 80000 Yéménites, marins pour l'essentiel, séjournaient en Angleterre dans les années 1870, leur circulation étant beaucoup plus aisée depuis l'ouverture du Canal de Suez en 1869.

6 Les Sikhs sont aujourd'hui un peu plus de 20 millions dans le monde (dont quelque 19 millions en Inde). La plus grosse communauté sikhe en dehors de l'Inde vit au Royaume-Uni, avec, selon le recensement de 2001, 336179 représentants $^{7}, 389000$ selon une estimation de novembre $2008 .^{8}$ Le Sikhisme, religion syncrétique dérivée de l'Hindouisme et de l'Islam, mais aussi de diverses mouvances philosophiques ou spirituelles indiennes, a pour berceau historique le Pendjab (où vivent 14 millions et demi des Sikhs indiens), d'où est issue la quasi-totalité des Sikhs de la diaspora présente au Royaume-Uni, au Canada, aux États-Unis, en Malaisie, en Thaillande, Singapour ou Hong Kong. C'est un prédicateur, Nanak, qui fonda le sikhisme au début du XVI e siècle. Il créa le village de Kartarpur, entouré de sikhs (disciples). Le Sikhisme adopta la tradition martiale et égalitaire des jats, anciens éleveurs nomades sur les terres desquels de nombreux villages sikhs furent fondés. L'enseignement de Nanak reposait notamment sur la foi en un dieu unique, éternel, tout puissant, sans forme ni attribut. Après la mort de Nanak, neuf gurus dits «fondateurs " se succédèrent, chacun contribuant à l'édification du sikhisme: le premier successeur de Nanak, Angad, inventa l'écriture gurmukhi, dans laquelle sont écrits les textes religieux des Sikhs et avec laquelle ils écrivent le pendjabi. C'est le dernier guru, Gobind (1666-1708) qui institua « les cinq k », les préceptes les plus connus du sikhisme : barbe et cheveux non coupés (kes) et retenus par un peigne (kangha), une petite épée (kirpan), un bracelet de métal (kara) et une culotte courte (kaach). ${ }^{9}$ Le turban, quant à lui, constitue un enjeu symbolique, identitaire et politique primordial, 
mais ne fait toutefois pas partie, à proprement parler, des prescriptions du sikhisme. ${ }^{10} \mathrm{Il}$ existe, par ailleurs, un certain nombre de tabous séculaires, toujours en vigueur chez les Sikhs orthodoxes: consommation de tabac et de viande d'animaux tués selon le rite musulman, ainsi que rapport sexuels avec des femmes musulmanes. Les hommes sikhs ajoutèrent Singh («lion ») à leur patronyme, et les femmes, Kaur («princesse »). Ce sont ces mots qui permettent de distinguer les noms des hommes et des femmes, dans la mesure où, dans ce groupe religieux, il n'existe pas de prénoms masculins ou féminins.

7 Loyaux à la Couronne lors de la Révolte des Cipayes (1857), les Sikhs bénéficièrent d'un recrutement préférentiel dans l'armée des Indes. Cette fidélité leur valut l'animosité durable des hindous et musulmans, mais elle créa, selon Gurharpal Singh, un « lien anglo sikh $»^{11}$ privilégié, fondé sur le respect mutuel. Si la réalité de cette relation est sujette à caution $^{12}$, elle fut longtemps inculquée aux jeunes Britanniques, comme en témoigne ce passage, extrait d'un manuel à la gloire des forces armées britanniques destiné aux enfants et largement diffusé dans le premier quart du XX siècle, The Wonder Book of Soldiers for Boys and Girls :

It is a mistake to suppose that when the tragedy of the Mutiny occurred, every native regiment sided with the rebels. [...] Our chief help, perhaps, came from the Frontier and Punjab battalions; and also from the Sikh troops. Indeed, but for their devoted assistance in the dark days of 57 , it is doubtful if the British flag would still be flying in India, as the white soldiers were immensely outnumbered by the followers of Nana Sahib ${ }^{13}$.

Le Maharajah Duleep Singh, dernier souverain du royaume sikh du royaume du Pendjab chassé du pouvoir par la East India Company, fut le premier Sikh à s'installer outreManche, en 1849, même s'il s'était converti au christianisme avant son arrivée en Angleterre. Dunleep Singh, surnommé le "Prince noir", percevait une pension très conséquente de l'India Office et menait grand train à Elveden Hall, domaine du Suffolk dont il fit l'acquisition en 1863, où il côtoyait aristocrates et capitaines d'industrie. Il envisagea même un temps de se présenter à la Chambre des Communes, avant d'en être dissuadé par la Reine Victoria elle-même ${ }^{14}$.

9 Sans surprise, la construction et le contrôle des gurdwaras et des mosquées allaient figurer parmi les enjeux initiaux des visibilités sikhe et musulmane dans la seconde moitié du $\mathrm{XX}^{\mathrm{e}}$ siècle $^{15}$, période au cours de laquelle on vit véritablement Sikhs et musulmans s'installer et s'organiser outre-Manche, selon des modalités tantôt comparables, tantôt différentes.

\section{Mosquées et gurdwaras}

10 À l'inverse des Sikhs, qui constituaient un groupe clairement identifié (bien qu'à de nombreux égards méconnu) depuis l'époque coloniale, les musulmans en tant que tels n'étaient pas perçus comme un groupe distinct. À de rares exceptions près ${ }^{16}$, les sciences sociales les considéraient au premier chef comme des Indiens, Pakistanais et, plus tard, Bangladais ou, encore, « Asiatiques » (Asians).

11 En ce qui concerne les lieux de culte, on assista à l'ouverture de mosquées et gurdwaras «pionnières" bien avant les débuts de l'immigration de masse. L'aménagement des premières «mosquées " (le plus souvent dans des maisons particulières, comme ce fut le cas à Cardiff, où la première "mosquée » du pays ouvrit en 1860) fut motivé par la présence d'une forte communauté yéménite dans le dernier tiers de l'ère victorienne, 
mais, également, par l'initiative d'une poignée de convertis, comme Henry Quilliam, qui fonda le Liverpool Muslim Institute en 1889. La même année, à Woking, était inaugurée la première mosquée construite à dessein sur des plans de l'orientaliste d'origine hongroise Gottlieb Wilhem Meitner, financée par des mécènes indo-musulmans. Pendant la Première Guerre mondiale, c'est à proximité de cette mosquée qu'allait être installé le premier cimetière militaire musulman, afin d'inhumer les milliers de soldats de l'Empire morts au combat. ${ }^{17}$ Pendant la Deuxième Guerre, en 1941, la mosquée de l'East End fut inaugurée à Londres, même si contrairement à la Grande Mosquée de Paris, inaugurée en 1926, elle n'avait pas ni le statut, ni la stature d'une grande mosquée centrale ${ }^{18}$.

12 Au tournant du XXe siècle, nombre de jeunes Indiens des classes aisées séjournaient à Londres, dont quelques Sikhs, qui fondèrent en 1908 la première association sikhe du royaume, Khalsa Jatha of the British Isles (KJBI) affiliée à la Chief Khalsa Divan (CKD), basée à Amritsar et alors principale association sikhe du Pendjab. Les Sikhs de la KJBI levèrent des fonds, en Angleterre et en Inde, afin de fonder une gurdwara à Londres. Une maison fut d'abord louée à Putney en 1911, avant l'achat d'un immeuble situé Sinclair Road, dans le quartier de Shepherd's Bush, qui après une modification architecturale totale, devint en 1913 la première véritable gurdwara du pays ${ }^{19}$. Traditionnellement, la gurdwara joue un rôle politique fondamental au Pendjab, où fut fondé dans les années 1920 le SGPC ( Shiromani Gurwara Prabandak Committee), instance chargée de gérer les gurdwaras, souvent surnommée le « Parlement sikh » et dotée d'une aile politique, le SAD (Shiromani Akali Dali ), principal parti politique sikh du Pendjab depuis 1925.

Ces lieux de culte pionniers, bien que hautement symboliques, ne doivent pas occulter le fait que pendant plusieurs décennies, les deux groupes religieux utilisèrent pour l'essentiel des lieux de prière informels, notamment dans des maisons particulières. Olivier Esteves fait remarquer que le manque de moyens n'était pas la seule raison à cela : les immigrés pakistanais ou indo-musulmans, souvent seuls en Grande-Bretagne, percevaient leur présence comme temporaire et ne voyaient pas la nécessité de construire des mosquées, qui de toutes les manières, au-delà de considérations financières et de l'attitude souvent hostile des autorités à l'égard de tels projets, auraient été disproportionnées compte tenu du nombre de fidèles potentiels ${ }^{20}$.

De la même manière, Singh et Singh Tatla observent que, en raison du nombre encore réduit de Sikhs, la gurdwara de Shepherd's Bush demeura la seule du pays jusqu'au début des années 1950. Comme chez les musulmans, les lieux de culte informels dominèrent les années 1950 et le début des années 1960 (malgré l'aménagement, voire, la construction de quelques gurdwaras, comme ce fut le cas à Bristol, dans le quartier de Easton, dès 1958). L'accélération de l'immigration en raison de l'imminence des restrictions induites par le Commonwealth Immigrants Act de $1962^{21}$, conjuguée au développement du regroupement familial puis à l'arrivée de Sikhs chassés des anciennes colonies d'Afrique de l'Est en vertu des politiques dites "d'indigénisation ", décuplèrent en vingt ans le nombre de Sikhs présents outre-Manche (de 7000 en 1951 à 72000 en 197122) et créèrent donc de nouveaux besoins en termes de lieux de culte dans les localités ou quartiers où ils étaient les plus représentés, les « petits Pendjabs » : Slough, Hounslow, Ealing (Southall), Wolverhampton ou encore, Gravesham, par exemple ${ }^{23}$.

La deuxième moitié des années 1960 et les années 1970 furent caractérisées par l'achat d'immeubles plus conséquents, le plus souvent délabrés, situés dans des inner cities dans lesquels (ou en lieu et place desquels) furent installées des gurdwaras. Dans les années 1980, les gurdwaras existantes allaient fréquemment être modifiées ou agrandies (en 1981, 
le nombre de Sikhs avaient doublé en l'espace de dix ans, passant à 144000$)^{24}$. Par ailleurs, sous l'impulsion de factions dissidentes mues par des rivalités de caste, on commençait à assister à l'apparition de nouveaux lieux de culte. Depuis les années 1990, la tendance est à la construction de gurdwaras beaucoup plus grandes, comme la Sri Guru Singh Sabha Gurdwara, (SGSSGS), inaugurée dans le quartier de Southall à Londres, en 2003. D'une capacité de trois mille fidèles, elle est la plus grande gurdwara en dehors de l'Inde ${ }^{25}$. En 2006, il existait deux cent quatorze gurdwaras au Royaume Uni (cent quatre-vingt-dixneuf en Angleterre, trois au Pays de Galles, onze en Ecosse et une en Irlande du Nord), contre une seule en 1951.

Sans surprise, chez les musulmans pakistanais et indiens, c'est également la perspective de la fin de l'immigration de masse, au tournant des années 1960 et le développement du regroupement familial par la suite qui sont à l'origine de l'augmentation du nombre de fidèles et donc, de mosquées ${ }^{26}$. L'arrivée des East African Asians dans les années 1960 et 1970, quant à elle, a eu un impact démographique bien moindre que pour les Sikhs et les Hindous, car les populations originaires du sous-continent indien installées au Kenya, en Ouganda, en Tanzanie ou au Malawi comptaient peu de musulmans. Comme pour les gurdwaras, l'installation des mosquées, de taille généralement beaucoup plus conséquente que les lieux de culte pionniers mentionnés plus haut s'accéléra dans les années 1970 : Manchester (1971-1972), Birmingham (1975), Londres (Mosquée centrale de Regent's Park, 1977) etc. ${ }^{27}$.

Il est à noter qu'à l'instar des gurdwaras, les mosquées (qui hébergeaient nombre d'associations cultu(r)elles) étaient souvent construites ou aménagées dans des inner cities où immeubles et terrains étaient relativement accessibles et où, en outre, vivaient de nombreux immigrés. Dans ces quartiers, lieux de culte musulmans et sikhs avaient parfois été précédés par des synagogues construites dans les années 1930, lorsque s'y installèrent des Juifs d'Europe centrale et orientale qui fuyaient les persécutions antisémites : ce fut le cas à Easton, inner city de Bristol, où une synagogue fondée dans l'entre-deux-guerres côtoie mosquées et gurdwara. Ce schéma a prévalu dans de nombreuses villes et le nombre de mosquées est estimé aujourd'hui à $1595^{28}$, contre 600 en $1990^{29}$.

\section{Inhumation}

Parmi les autres demandes exprimées assez tôt par les immigrés de confession musulmane figurait en bonne place la prise en compte des spécificités de l'inhumation dans l'islam (ensevelissement dans les vingt-quatre heures suivant le décès, orientation de la tête du défunt vers la Mecque, absence de cercueil, notamment) à une époque où le «mythe du retour », encore vivace dans les années 1970, s'estompait peu à peu ${ }^{30}$ et où, par conséquent, commençait à se poser la question de l'enterrement des musulmans au Royaume-Uni. Si cette préoccupation émergea vraiment dans les années 1980 avant de prendre une ampleur inédite dans les années 1990, elle s'était néanmoins ponctuellement posée avant et pendant la Seconde Guerre mondiale. En dehors du cimetière attenant à la mosquée de Woking, le premier carré musulman fut créé en 1937 à South Shields ${ }^{31}$, non loin de Newcastle-upon-Tyne. Cinq ans plus tard, à Birmingham, une Anglaise mariée à un musulman indien, Mary Amirullah, fit campagne pour la création d'un carré musulman, au sein du cimetière de Lodge Hill, demande à laquelle la municipalité accéda en 1942, Jusqu'alors, dans les cimetières paroissiaux, les rares musulmans étaient enterrés, comme tous les autres non-anglicans, dans le carré réservé aux non-conformistes. Dans les 
cimetières municipaux, ils l'étaient dans la section générale mais sans aucun signe distinctif $^{32}$.

La question de l'inhumation ne se posa pas pour les Sikhs, en raison de la pratique généralisée de la crémation (proscrite chez les musulmans) et de la dispersion des cendres, le plus souvent dans des rivières ou dans la mer, mais aussi, parfois, dans un lieu auquel le défunt était attaché33. La crémation sur des bûchers funéraires à ciel ouvert, qui est la norme pour les Sikhs (et les Hindous) en Inde est interdite au Royaume Uni, en vertu du Cremation Act de 1902. Bien que rares, des entorses à la loi ont été commises, comme en 2006, lorsque la famille de Rajpal Mehat avait procédé dans l'illégalité à sa crémation ${ }^{34}$. Néanmoins, une décision de la Court of Appeal rendue en février 2010 risque de faire jurisprudence, puisque ce tribunal a reconnu à Davender Ghai, leader religieux hindou, le droit d'être incinéré sur un bûcher à ciel ouvert ${ }^{35}$. Cette décision ouvre donc la voie à de nouvelles demandes similaires, bien qu'elles demeurent marginales et que la plupart des incinérations de Sikhs (et d'Hindous) aient lieu dans des crématoriums classiques.

La prochaine partie portera sur les avancées dont musulmans et Sikhs ont bénéficié au cours des dernières décennies. Elle comparera l'affirmation identitaire et la construction communautaire de ces deux groupes religieux et analysera l'impact des rapports avec la société dominante dans ce processus.

\section{Modalités de mobilisation communautaire et reconnaissance publique Les affaires Rushdie \& Behzti}

21 Dans les années 1980, on assista à un frémissement dans l'apparition d'associations musulmanes, qui, fréquemment, constituaient les branches de mouvements transnationaux, implantés dans les pays musulmans ou dans des pays occidentaux où vivaient désormais d'importantes populations musulmanes. Parmi elles, on pourra citer Young Muslims (1984), émanation d'un mouvement transnational qui lança une association équivalente en France en 1987 (Jeunes Musulmans de France) et Hizb ut-Tahrir (1986), association controversée et teintée de radicalisme, branche du mouvement éponyme fondé à Jérusalem en 1953.

Ces associations apparurent surtout à partir du milieu des années 1980, au terme d'une période marquée par les émeutes urbaines de l'ère Thatcher, qui s'étalèrent de 1980 à $1985^{36}$. Ces incidents, qui impliquèrent essentiellement de jeunes Noirs originaires de la Caraïbe, servirent de caisse de résonnance aux revendications culturelles jusqu'alors peu coordonnées de ce groupe et signala l'entrée dans le "multiculturalisme ", transition facilitée par l'existence d'une matrice différentialiste mise en place par les travaillistes dans les années 1960 et $1970^{37}$.

Quasi absents des émeutes urbaines du début et du milieu des années 1980, les Pakistanais, Bangladais et Indiens musulmans eurent, eux aussi, leur crise structurante, qui les fit émerger en tant que "musulmans »: l'affaire Rushdie. Cette crise eut une dimension internationale et même, transnationale. Elle éclata en 1988 avec la publication de Versets sataniques et culmina en février 1989 avec la fatwa de Khomeiny, le leader iranien, appelant à l'exécution de l'auteur anglo-indien. Le refus du gouvernement Thatcher d'interdire les Versets sataniques et celui de la Chambre des Lords d'appliquer à 
l'islam ${ }^{38}$ les lois séculaires sur le blasphème, radicalisa une partie des musulmans britanniques, qui accepta la fatwa et, peu à peu, s'érigea en communauté ${ }^{39}$, même si Olivier Esteves observe qu'une majorité de musulmans fut tout autant choquée par l'ouvrage de Rushdie que par la fatwa de Khomeiny ${ }^{40}$.

L'affaire Rushdie conféra aux musulmans britanniques une visibilité jusqu'alors inédite et en fit une menace aux yeux de l'opinion, qui pour la première fois vraiment, commença à les percevoir en tant que groupe religieux distinct (ce qui était déjà le cas des Sikhs, et, dans une moindre mesure, des Hindous). Du jour au lendemain, Pakistanais et Bangladais devinrent non seulement des musulmans, mais aussi, pour une bonne partie de l'opinion, des extrémistes. Une partie de la mobilisation et de l'action collective nées de la publication des Versets sataniques prit d'emblée une tournure assez radicale, certes minoritaire mais amplement relayée dans les médias, comme les propos désastreux de Iqbal Sacranie (leader national qui émergea lors de cette crise), qui semblaient justifier la condamnation à mort de Salman Rushdie ${ }^{41}$. Un autodafé eut lieu dans les rues de Bradford en janvier 1989, un mois avant la fatwa. Les images de cet événement eurent, là aussi, un effet calamiteux, en créant des tensions dans le monde musulman, et en convainquant nombre de Britanniques de la dangerosité d'un groupe jusqu'alors invisible et désormais réduit à son identité religieuse supposée. Les associations musulmanes laïques ou interconfessionnelles qui condamnèrent ouvertement la fatwa et défendirent la liberté d'expression ${ }^{42}$ furent pour l'essentiel ignorées par les grands médias nationaux qui cédèrent au sensationnalisme. La crise contribua à la notoriété d'associations telles que the Bradford Council of Mosques ${ }^{43}$, une des premières structures qui chercha à mobiliser les musulmans en tant que groupe spécifique, à l'instar du Islamic Party of Britain, parti politique musulman créé en 1989 et dont l'impact fut néanmoins minime.

Les centaines d'associations, le plus souvent locales et de petite taille, créées en réaction à l'affaire Rushdie cherchèrent à se fédérer. Désunies et novices en politique, les associations existantes au début de la crise n'avaient su faire suffisamment pression sur les pouvoirs publics pour obtenir l'interdiction de l'ouvrage controversé. C'est à cet effet que des structures temporaires furent créées, regroupant des associations le plus souvent très conservatrices et des militants très majoritairement originaires du sous-continent indien : UKACIA (United Kingdom Action Committee on Islamic Affairs) créée fin 1988, au tout début de la crise puis le NICMU (National Interim Committee on Muslim Unity), en 1994 et qui deux ans après décida de la création d'une grande instance représentative, le Muslim Council of Britain (MCB), officiellement lancée en 1997 avec le soutien du gouvernement britannique, qui ne lui accorda toutefois pas de monopole sur la représentation de l'islam ${ }^{44}$.

Même il ne s'agit évidemment pas de réduire la visibilité et l'affirmation identitaire musulmanes à la seule affaire Rushdie ou de nier l'impact d'événements ultérieurs (Guerres du Golfe, de Bosnie, d'Irak et d'Afghanistan, caricatures de Mahomet), il demeure que la crise suscitée par la publication des Versets sataniques fut de loin la plus structurante, puisque c'est celle qui donna corps à une "communauté " (même si son homogénéité est illusoire) jusqu'alors faiblement identifiée et constituée. De nombreux commentateurs ont dressé un parallèle entre la crise suscitée par les Versets sataniques et celle déclenchée en 2004, en plein débat sur le rôle de la religion dans la vie publique par la pièce de théâtre Behzti (déshonneur, en pendjabi), souvent qualifiée d'« affaire Rushdie sikhe $»^{45}$, même si ces deux affaires intervinrent à des moments très différents dans la structuration des deux communautés. L'auteure de Behzti, Gurpreet Kaur Bhatti, sikhe 
elle-même, avait placé l'action de sa pièce dans une gurdwara, théâtre d'aventures homosexuelles, viols et finalement, d'un meurtre perpétré à l'aide d'un kirpan, la petite épée rituelle sikhe. Avant la première représentation, des représentants du Conseil des gurdwaras de Birmingham firent part de leur inquiétude au Repertory théâtre de Birmingham où la pièce devait être jouée. Conviés à une lecture de la pièce, ils demandèrent à ce que l'action ne se déroule pas dans une gurdwara, mais dans un centre communautaire. La direction du théâtre n'accéda pas à cette demande, qui, selon elle, s'apparentait à une tentative de censure. Le soir de la première, le 18 décembre 2004, des échauffourées eurent lieu en marge d'une manifestation organisée à l'appel des associations sikhes de la ville et regroupant 400 personnes. Un groupe s'introduisit dans le théâtre, obligeant l'évacuation de 800 personnes (des familles, pour l'essentiel), qui assistaient à un spectacle de Noël. Trois policiers furent blessés et trois manifestants, interpelés. Le théâtre décida de déprogrammer les représentations, tandis que Gurpreet Kaur Bhatti, menacée, dut se cacher et être placée momentanément sous la protection de la police, comme Rusdhie quinze années auparavant. Fiona MacTaggart, ministre du gouvernement Blair et députée de Slough, refusa de critiquer la violence, tout en soulignant que la manifestation constituait « un signe de liberté d'expression, qui fait éminemment partie de la tradition britannique $»^{46}$. Presse et médias audiovisuels s'emparèrent de l'affaire, provoquant un nouveau débat sur la liberté d'expression. De nombreux artistes et intellectuels exprimèrent leur soutien à la dramaturge dans les colonnes du Guardian. ${ }^{47}$ Salman Rushdie lui-même critiqua la déprogrammation de la pièce, ainsi que la déclaration de Fiona MacTaggart, emblématique, selon lui, de l'ambivalence du New Labour à l'égard de la liberté d'expression, alors que les conservateurs l'avaient soutenu au moment de l'affaire Rushdie ${ }^{48}$.

Néanmoins, la comparaison entre les deux affaires a ses limites. En effet, une lecture moins superficielle des modes de mobilisation des deux groupes religieux révèle que le caractère soudain et relativement unique de l'affaire Rushdie (qui se solda par un échec) se distingue des crises multiples qui ont opposé les Sikhs à la société dominante et dont la campagne victorieuse contre Behzti a été un des derniers avatars. Les paragraphes suivants proposeront une lecture à rebours de cette lutte des Sikhs pour la visibilité et la reconnaissance. Ils en analyseront les principaux épisodes et comporteront des éléments de comparaison avec les musulmans lorsque cela sera pertinent.

\section{Des crises à répétition et un fonctionnement par à- coups : mode d'affirmation identitaire des Sikhs}

Bien avant les Antillais et les musulmans, pourtant bien plus nombreux qu'eux, et même, bien avant le tournant des années 1980, qui signala l'entrée dans le multiculturalisme à proprement parler, les Sikhs surent se mobiliser et faire pression sur les autorités afin d'affirmer leur identité. Cette mobilisation fut essentiellement le fait des Sikhs kesh-dari, c'est-à-dire, ceux qui ne se coupent ni cheveux ni barbe et portent le turban. C'est précisément au nom du droit à porter le turban que quatre campagnes s'enchaînèrent sur trois décennies.

La première campagne des turbans s'étala sur dix ans au total, à Manchester (1959-1966) puis Wolverhampton (1967-1969). À l'origine de la première phase cette campagne, le 
refus de la régie municipale des transports de Manchester de recruter un contrôleur sikh, G.S. Sagar, en raison du turban qu'il portait. La toute récente gurdwara de Manchester le soutint et mena une campagne de longue haleine (appels au boycott des bus de la ville, manifestations etc.). Finalement, après d'âpres débats, le Council de Manchester reconnut le droit des Sikhs à porter leur turban dans l'exercice de leurs fonctions en 1966, un an après l'adoption du premier Race Relations Act et l'année même où Roy Jenkins, Home Secretary de Harold Wilson, donna sa définition de l'intégration, rejetant toute tentative $d^{\prime}$ '« uniformisation » culturelle ${ }^{49}$.

La deuxième phase de cette campagne eut lieu dans les Midlands en 1967, à Wolverhampton, dont une des circonscriptions, Wolverhampton South West, était alors celle de Enoch Powell. Tarsem Singh Sandhu, chauffeur de bus sikh qui avait les cheveux courts lors de son recrutement par le Council, revint au travail coiffé d'un turban au terme d'un congé maladie, ce qui lui valut d'être licencié pour violation du code vestimentaire de la compagnie. La campagne qu'il lança en réaction à cette décision, revêtit une dimension locale, nationale et même, transnationale. Locale car, ayant manifestement mal jaugé le personnage, la Indian Workers'Association de Wolverhampton sollicita, en vain, Enoch Powell afin qu'il intervienne auprès de la municipalité5 ${ }^{5}$. Nationale, car dans son célèbre discours apocalyptique de 1968, le ténor conservateur fit spécifiquement référence aux revendications culturelles sikhes, en citant, pour apporter de l'eau à son moulin, les propos du travailliste John Stonehouse, alors ministre du gouvernement Wilson:

'The Sikh communities' campaign to maintain customs inappropriate in Britain is much to be regretted. Working in Britain, particularly in the public services, they should be prepared to accept the terms and conditions of their employment. To claim special communal rights (or should one say rites?) leads to a dangerous fragmentation within society. This communalism is a canker; whether practised by one colour or another it is to be strongly condemned.' All credit to John Stonehouse for having had the insight to perceive that, and the courage to say it ${ }^{51}$.

Transnationale, car à l'issue de deux marches pacifiques qui rassemblèrent 5000 Sikhs à Wolverhampton, un leader communautaire local, Sohan Singh Jolly, menaça de s'immoler par le feu, le 13 avril 1969, deux ans après le début du différend. Cette menace suscita l'intervention du Haut-Commissaire indien, qui alerta le Council et le gouvernement des répercussions que ce suicide pourrait avoir en Inde. Suite à cette intervention, les règles furent changées, quatre jours avant l'expiration de l'ultimatum de Singh Jolly.

Cette victoire sembla remise en question par l'entrée en vigueur, le $1^{\mathrm{er}}$ juin 1973, d'une loi rendant obligatoire le port du casque pour les motocyclistes (Section 32 du Road Traffic Act de 1972), ce qui obligeait donc les motards sikhs à ôter leur turban et donna lieu à la deuxième campagne des turbans (1973-1976). Après la fin de non-recevoir opposée à une délégation reçue au Ministère $d u$ Transport, la campagne monta en puissance, un candidat sikh se présentant même à Ealing lors de l'élection générale de 1974, avec pour tout programme l'obtention d'une dérogation pour ses coreligionnaires. Ce candidat, Baldev Singh Chahal, avait préalablement saisi la High Court, qui avait confirmé que les Sikhs étaient bien soumis à la loi, concluant en substance que c'était leur religion et non pas la loi britannique qui les empêchait de conduire une moto. La Cour Européenne des Droits de l'Homme, également saisie, conclut que l'interférence éventuelle avec la liberté religieuse était justifiée par des impératifs de sécurité et de santé52.

33 Le gouvernement travailliste de Harold Wilson finit par accorder cette exemption, avec le Motor-Cycle Crash Helmets (Religious exemption) Act en 1976, année de l'adoption du 
troisième Race Relations Act. Elle fut par la suite confirmée sous les conservateurs, en 1988 (Road Traffic Act). Ce débat, bien que largement symbolique compte tenu du nombre très limité de motocyclistes parmi les Sikhs kesh-dari, eut un écho important et illustrait bien les clivages émergents au sein de la société britannique au sujet du « multiculturalisme ", même si le terme n'avait pas encore cours outre-Manche. Pour protester contre la dérogation accordée aux Sikhs deux motards « chrétiens » se coiffèrent d'un turban pour piloter leur deux-roues, l'un d'entre eux étant même coiffé de ce couvre-chef lors de son procès ${ }^{53}$.

La troisième campagne des turbans débuta en 1978 et se conclut en 1983 par une décision de justice décisive dans le processus de construction communautaire des Sikhs de Grande-Bretagne, et, au-delà, à travers le monde. Un élève sikh, Gurinder Singh Mandla qui souhaitait poursuivre sa scolarité dans un établissement privé de Birmingham, Park Grove School, vit sa candidature rejetée, car son turban contrevenait aux règles de l'école en matière d'uniforme. Le père de l'adolescent porta plainte pour discrimination raciale auprès de la Commission for Racial Equality. L'instance utilisa cette affaire pour tester la notion de discrimination indirecte ${ }^{54}$, concept identifié deux ans auparavant par le Race Relations Act de 1976. En première instance, la county court rejeta la plainte au motif que le port du turban ne constituait qu'une pratique religieuse, étrangère donc à toute discrimination raciale ${ }^{55}$. Cette décision fut confirmée en 1982 par la Cour d'appel, dont le Président tourna en ridicule la plainte déposée :

The right not to be discriminated against must give way to the beliefs and free will of others. If persons wish to insist on wearing bathing suits, they cannot reasonably insist on admission to a nudist colony; similarly people who passionately believe in nudism cannot complain if they are not accepted on ordinary bathing beaches ${ }^{56}$.

L'affaire Mandla mobilisa énormément au Royaume-Uni, où se déroulèrent de nombreuses manifestations à Birmingham et Londres, dont une de 40000 personnes à Hyde Park, en octobre 1982. Elle mobilisa également au Pendjab, où le SAD, principal parti sikh qui faisait alors campagne pour une plus grande autonomie politique des Sikhs, en fit un symbole. La Commission for Racial Equality et la famille Mandla saisirent la Chambre des Lords, qui, le 23 mars 1983, donna raison aux plaignants et reconnut aux Sikhs ${ }^{57}$ le statut de groupe ethnique, en raison d'une longue histoire commune, qui les distingue des autres groupes et d'une tradition culturelle spécifique, souvent mais pas exclusivement associée à la pratique religieuse. Cette décision historique conférait donc aux Sikhs, à l'instar des Juifs, le statut double de minorité religieuse et raciale, reconnu depuis à aucun autre groupe religieux, hindous et musulmans compris. Cette victoire allait ouvrir aux Sikhs de nouveaux droits et exemptions, même si clairement la lutte se poursuivait, conformément à la stratégie de l'à-coup et de la victoire symbolique.

La dernière campagne des turbans à ce jour s'est achevée à la fin de l'ère Thatcher, lorsque par le biais de la Section 11 du Employment Act de 1989 le gouvernement accorda aux Sikhs employés du secteur de la construction une dérogation relative au port du casque de chantier, obligatoire depuis $1979^{58}$. Cette concession, qui intervint en pleine affaire Rushdie, fit suite à une campagne très habilement menée par la British Sikh Association dans les gurdwaras et la presse pendjabie. Lorsqu'elle fut remise en cause par une directive européenne sur le port obligatoire de casques de chantiers par les travailleurs censée entrer en vigueur en 1992, le Royaume-Uni négocia une exception, qui ne s'appliquait qu'aux travailleurs du bâtiment, en raison du nombre important de Sikhs dans ce secteur (40 000 environ, à l'époque) ${ }^{59}$. 


\section{Place des Sikhs et des musulmans dans le système scolaire}

37 L'issue de Mandla Vs Dowell Lee avait montré que la place des Sikhs dans le système scolaire constituait un élément moteur dans leur affirmation identitaire et leur reconnaissance par la société dominante. Comme nous allons le voir, l'école continue à jouer ce rôle, pour les Sikhs, mais aussi pour les musulmans. Si l'immense majorité des Sikhs fréquentent des écoles d'État (et pour certains, des écoles privées ou sous-contrat non-sikhes) il existe aussi plusieurs écoles sikhes privées, notamment dans les Midlands et dans la région de Londres. En 1999, l'une d'entre elles, Guru Nanak Sikh College, à Hillingdon, Londres, fondée en 1993, fut la première à recevoir des financements publics, avant d'être rejointe en 2006 par un établissement de Slough (localité qui, avec ses 10000 Sikhs, fait partie des « Petits Pendjabs »).

Les écoles musulmanes privées, quant à elles, ont vu leur nombre fortement augmenter dans les années 1990 et 2000, même si in fine, les 115 écoles recensées n'accueillent qu'1 \% des élèves de confession musulmane ${ }^{60}$. Les premières demandes de financement public furent formulées par des établissements musulmans à la fin des années 1980. Le gouvernement Blair accéda aux deux premières demandes en 1998. On compte aujourd'hui huit établissements musulmans "sous contrat", l'apparition d'établissements confessionnels que Tony Blair entendait un temps promouvoir ayant été sérieusement contrariée par les heurts interethniques de l'été 2001, les attentats du 11 septembre et le virage « intégrationniste » qui les a suivis.

La question du code vestimentaire de certains musulmans a suscité moins de controverses chez les garçons musulmans que chez leurs homologues sikhs, dans la mesure où, a priori ils sont moins concernés par le port de signes religieux. Ponctuellement, néanmoins, le port d'une barbe fournie chez des garçons de 17-18 ans a pu être controversé dans certains établissements. La question du voile islamique pour les filles n'est pas jugée problématique en soi, mais, au cours des dernières années, des mesures ont été prises pour autoriser les chefs d'établissement à interdire le port de voiles intégraux ${ }^{61}$ (ou, du moins, très couvrants) et on a assisté à des exclusions définitives d'élèves ou à des licenciements d'enseignantes pour ce motif ${ }^{62}$. Cette fermeté relative ne doit cependant pas occulter le fait que des aménagements, parfois très significatifs, ont été mis en place au cours des dernières années afin d'aider les élèves musulmans qui le souhaitent à mieux vivre leur foi au sein des écoles d'État. En 2007, le Muslim Council of Britain, principale instance représentative musulmane, a publié un guide destiné aux chefs d'établissement, recommandant, entre autres, que le port du voile islamique ou de la barbe soit toléré, que filles et garçons soient autorisés à porter un survêtement plutôt qu'un short en cours d'éducation physique etc. Ce fascicule préconisait également l'aménagement de salles de prière, y compris de salles non-mixtes si des élèves, leurs parents ou des enseignants musulmans en formulaient la demande ${ }^{63}$. De nombreux établissements ont accédé à de telles revendications, même lorsqu'elles étaient marginales, comme Fairfield High, par exemple, à Bristol, qui, en outre, a décidé de relayer dans l'enceinte de l'établissement les appels à la prière du vendredi pendant la période de Ramadan ${ }^{64}$, en contradiction flagrante tant avec les objectifs de la community cohesion, qu'avec la fermeté affichée par ailleurs envers d'autres pratiques culturelles ou coutumes musulmanes. 
Malgré les victoires acquises par les Sikhs à la faveur des diverses campagnes des turbans, l'autorisation sous certaines conditions du port du kirpan ${ }^{65}$ dans les écoles britanniques peut légitimement laisser perplexe, surtout au vu du débat de ces dernière années sur la présence inquiétante de couteaux dans les établissements scolaires outre-Manche ${ }^{66}$ et le projet du gouvernement Brown, finalement peu appliqué, d'installer des détecteurs de métaux à l'entrée des écoles ${ }^{67}$. Cette exemption surprenante, justifiée au nom du Race Relations (Amendment) Act de 2000, est laissée à la discrétion du chef d'établissement, qui doit rencontrer les élèves concernés et leurs parents et leur conseiller le port d'un kirpan symbolique, en plastique. En cas de refus, le port d'un kirpan métallique à bout arrondi est proposé. Si la famille s'y oppose, un vrai kirpan est autorisé à titre dérogatoire et sous diverses conditions. La taille et le type du kirpan est alors déterminée entre le chef d'établissement et la famille, le maximum autorisé étant de 8 pouces (soit $20,32 \mathrm{~cm}$ ), lame incluse. Il est à noter que ce port n'est autorisé que pour les Sikhs dits baptisés, les amritdhari, comme en atteste le texte suivant, daté de juin 2010 et émanant de l'autorité locale de Milton Keynes, dans le Buckinghamshire :

If a Kirpan is worn in school it should be:

- restricted to pupils who have gone through the Amrit Pahul ceremony;

- sheathed and secured, so that it cannot be withdrawn in school. This may be soldered or sewn into a purpose-made pouch;

- hidden at all times, worn under clothing and bound to the body with a long strap of fabric (kamarkassa) [...]

- securely locked up by the school if removed by a pupil for whatever reason. [...] $]^{68}$.

Bien que la distinction entre sphère publique et sphère privée, qui régit la place de la religion en France, soit infiniment plus perméable outre-Manche et que les établissements privés, sous contrat et même publics soient très majoritairement confessionnels, on ne peut être que très surpris que collèges ou lycées d'Etat exigent un " certificat de baptême " afin de valider l'autorisation de porter un kirpan, même si le port de cet attribut symbolique illustre une nouvelle fois le combat de longue haleine mené par les Sikhs pour la reconnaissance de leurs spécificités culturelles. En effet, aussi surprenant que cela puisse paraître, une exemption accordée aux employés d'aéroport pour leur permettre de porter le kirpan dans l'exercice de leur fonction par le gouvernement Thatcher en 1979 et confirmée en 1988 demeure également valable dans le contexte hyper-sécuritaire de l'après-11 septembre ${ }^{69}$, malgré le tollé qu'une telle dérogation a suscité au sein des syndicats de pilotes de ligne.

\section{Conclusion}

$\mathrm{Au}$ vu de cet état des lieux partiel et des pistes de comparaison ébauchées dans cet article, il apparaît que chacune des communautés a réussi un tour de force : les musulmans, celui d'émerger en tant que communauté en l'espace de quelques années, voire quelques mois à peine, même si cette visibilité s'est effectuée au prix d'une stigmatisation et d'une suspicion durables; les Sikhs celui, très paradoxal, de devenir de plus en plus visibles du point de vue ethnique et religieux, tout en restant, dans une grande mesure, invisibles aux yeux de l'opinion et, pour l'essentiel, absents du discours politique contemporain critique à l'égard du multiculturalisme, qui tend à se focaliser exclusivement sur la place de l'islam et des musulmans au Royaume-Uni et à ignorer la question du communautarisme sikh, pourtant pas toujours exempt de dérapages, comme lors de l'affaire Behzti en 2004. 
Même s'il ne s'agit pas de nier la réalité de certains aménagements dont ont pu bénéficier les musulmans, y compris dans le contexte de l'après-11 septembre, on a tout de même l'impression que les autorités britanniques jugent les revendications sikhes beaucoup moins problématiques que celles des musulmans. On peut légitimement se demander si, par exemple, la question du kirpan aurait été traitée de la même manière si cette petite épée avait été l'attribut d'une minorité de musulmans.

Quelles sont donc les raisons de cette différence d'attitude et de l'efficacité redoutable de l'action collective des Sikhs du Royaume-Uni?

Tout d'abord, le fameux lien séculaire anglo-sikh remontant à l'époque coloniale ne saurait être négligé, même s'il semble avoir été largement construit a posteriori. En deuxième lieu, l'homogénéité culturelle, linguistique et ethnique des Sikhs est sans commune mesure avec celle des musulmans et a également joué un rôle dans l'efficacité de l'action collective de ce groupe, même si les clivages et rivalités (notamment de castes) qui traversent les Sikhs du Royaume-Uni sont très souvent sous-estimés et trouvent d'ailleurs une illustration dans les difficultés rencontrées par les instances représentatives sikhes. En effet, les diverses tentatives pour créer une instance représentative relativement consensuelle et reconnue des pouvoirs publics a échoué. Si diverses fédérations (Sikh Human Rights Group, Network of Sikh Organisations, Sikh Secretariat ) affirment parler au nom de l'ensemble de la communauté, c'est le factionnalisme qui domine. À tout pendre, l'instance qui a établi les contacts les plus constants avec le gouvernement semble être la Sikh Federation, bien qu'elle n'ait jamais atteint l'influence et la relative légitimité dont put se prévaloir le Muslim Council of Britain sous les deux premiers mandats de Tony Blair (1997-2005), période où l'instance parvint à convaincre le gouvernement d'ajouter une question sur l'affiliation religieuse dans le recensement, alors que par contraste, à ce jour, les associations sikhes n'ont pu obtenir la création d'une catégorie ethnique "Sikh» qui leur permettrait de ne pas se définir comme «Indien ». Troisièmement, les Sikhs, bien que beaucoup moins nombreux que les musulmans (et que les Antillais) ont su d'emblée se mobiliser, sans doute parce que ce groupe, en Inde ${ }^{70}$ comme dans les anciennes colonies britanniques d'Afrique de l'Est où plusieurs dizaines de milliers s'étaient implantés à l'époque impériale, a toujours constitué une minorité, avec tous les réflexes de défense qu'un tel statut implique. À cet égard, on se souviendra par exemple des réactions de la minuscule communauté sikhe de France au moment de l'annonce puis de l'entrée en vigueur de la loi de 2004 sur les signes religieux dans les établissements scolaires publics. La stratégie des Sikhs outre-Manche est, comme nous l'avons vu, celle de la crise permanente et de l'à-coup, chaque victoire (dont la portée peut parfois passer pour symbolique, voire anecdotique, comme dans le cas des casques de moto) leur ayant permis d'affirmer leur identité et d'asseoir davantage leur présence au Royaume-Uni. La dernière raison découle directement de la précédente : il s'agit d'une communauté numériquement moins importante que la "communauté musulmane ", et donc, sans doute, moins anxiogène, surtout si l'on garde à l'esprit que la gestion d'une masse numérique problématique a été au cœur du couplage des politiques d'immigration avec des mesures d'intégration dès le début des années soixante et que la peur de voir le Royaume-Uni submergé par des populations et/ou des cultures différentes est une constante du discours xénophobe britannique, de Enoch Powell à Nick Griffin, en passant par Margaret Thatcher et Winston Churchill Junior. 


\section{NOTES}

1. En raison du format imparti, les questions socio-économiques, qui, à elles seules pourraient justifier un article entier, ne seront pas (ou très peu) abordées.

2. Focus on Religion, London: Office of National Statistics, 2004, [http://www.statistics.gov.uk/ downloads/theme_compendia/for2004/FocusonReligion.pdf] (lien actif le $1^{\text {er }}$ juin 2011).

3. Richard KERBAJ, "Muslim Population rising 'ten times faster than the rest of society'», The Times, 30 January 2009. [http://www.timesonline.co.uk/tol/news/uk/article5621482.ece]

4. Soit: $43 \%$ de Pakistanais / Britanniques d'origine pakistanaise; $16 \%$ de Bangladais / Britanniques d'origine bangladaise ; $8 \%$ d'Indiens / Britanniques d'origine indienne ; $6 \%$ d'autres «Asiatiques » (originaires notamment du Sri Lanka ou d'Afghanistan) Source : Focus on Religion, [ http://www.statistics.gov.uk/downloads/theme_compendia/for2004/FocusonReligion.pdf], p.5

5. [http://www.muslimsinbritain.org/resources/masjid_report.pdf] (lien actif le 20 mai 2011)

6. Comme en témoigne le somptueux Ismaili Centre de Londres (South Kensington) inauguré en 1985 par Margaret Thatcher.

7. On en compte 278000 au Canada, 250000 aux États-Unis, mais à peine 4000 en France. Source : Gurharpal SINGH \& Darshan SINGH TATLA, Sikhs in Britain: The Making of a Community, London / New York : Zed Books, 2006, p. 32.

8. Richard KERBAJ, "Muslim Population rising 'ten times faster than the rest of society' ", The Times, 30 January 2009. [http://www.timesonline.co.uk/tol/news/uk/article5621482.ece]

9. Denis MATRINGE, «Sikhs », CD-ROM Universalis Version 4, (Paris : Encyclopaedia Universalis, 1998).

10. SINGH \& SINGH TATLA, p. 127.

11. SINGH \& SINGH TATLA, p. 130.

12. Ainsi, en 1919, Akali Dal («Armée de l'Eternel»), une organisation prônant le retour aux sources du sikhisme, obtint une victoire importante contre les mahants, religieux corrompus à qui les Britanniques avaient confié la gestion de leurs temples. Beaucoup de Sikhs entrèrent dans le mouvement national, pris en tenaille entre un Congrès très majoritairement hindou et la Muslim League de Jinnah.

13. Harry GOLDING, The Wonder Book of Soldiers for Boys and Girls, third edition, London : Ward Lock \& Co Limited, 1922, p. 120.

14. SINGH \& SINGH TATLA, p. 44.

15. SINGH \& SINGH TATLA, p. 69.

16. Voir par exemple Patricia JEFFERY, Migrants and Refugees:Muslim and Christian Pakistani Families in Bristol, Cambridge : CUP, 1976.

17. Nada AFIOUNI, " Les cimetières britanniques à l'image du multiculturalisme britannique?" in Sexe, Race et Mixité, Michel PRUM (dir), Paris : L'Harmattan, collection "Racisme et eugénisme", 2011.

18. La Central Mosque de Londres n'allait être inaugurée qu'en 1977.

19. SINGH \& SINGH TATLA, p. 70.

20. Olivier ESTEVES, De l'invisibilité à l'islamophobie: les musulmans britanniques (1945-2010), Paris : Presses Universitaires de Sciences Po, 2011, p. 90.

21. On a alors de beat-the-ban immigration pour désigner cette course contre la montre.

22. Ceri PEACH \& Richard GALE, 'Muslims, Hindus and Sikhs in the New Religious Landscape of England', The Geographical Review, 93:2, 469-90, cité dans Singh \& Singh Tatla, p. 58

23. SINGH \& SINGH TATLA, p. 63. 
24. Ibid.

25. SINGH \& SINGH TATLA, p. 69.

26. Olivier ESTEVES, De l'invisibilité à l'islamophobie: les musulmans britanniques (1945-2010), op.cit., p. 91.

27. Ibid., p. 92

28. «UK Mosque Statistics», 16 March 2011 [http://www.muslimsinbritain.org/resources/ masjid_report.pdf] (lien accessible le 15 juin 2011).

29. Olivier ESTEVES, De l'invisibilité à l'islamophobie: les musulmans britanniques (1945-2010), op.cit., p. 93.

30. Ibid., pp. 69-85.

31. Nada AFIOUNI, «Les cimetières britanniques à l'image du multiculturalisme britannique? » in Sexe, Race et Mixité , Michel PRUM (dir), Paris, L'Harmattan, collection "Racisme et eugénisme", 2011.

32. Ibid.

33. «Commemorating death in Staffordshire: Sikh funerals » [http:// www.staffspasttrack.org.uk/exhibit/ilm/Mourining\%20and\%20Remembrance/Types\%20of\% 20funerals/Sikh\%20Funerals.htm] (lien acessible le 15 juin 2011)

34. Sam JONES, «Police say Sikh funeral pyre may have broken cremation laws ", The Guardian, Thursday 13 July 2006.

35. Jerome TAYLOR, "Hindu healer wins funeral pyre battle", The Independent, Wednesday, 10 February 2010. [http://www.independent.co.uk/news/uk/home-news/hindu-healer-winsfuneral-pyre-battle-1895116.html].

36. On pourrait y ajouter les émeutes de Southall, le 23 avril 1979, qui eurent lieu quelques jours avant l'élection de Margaret Thatcher (4 mai 1979), bien qu'elles aient eu une physionomie assez différente : heurts violents entre militants d'extrême droite et manifestants antiracistes dans un quartier où réside une des plus fortes concentrations de populations originaires du souscontinent indien du pays.

37. Pour plus de détail, voir Vincent LATOUR, «Les métamorphoses du multiculturalisme britannique ", Revue Française de Civilisation Britannique, Volume XIV $\mathrm{N}^{\circ} 3$, Le défi multiculturel en Grande- Bretagne, sous la direction de Gilbert MILLAT, Paris : Presses de la Sorbonne Nouvelle, décembre 2007.

38. Le délit de blasphème ne fut cependant aboli qu'en 2008, avec l'adoption du Criminal Justice and Immigration Act.

39. Anthony MC ROY, From Rushdie to 7/7. The Radicalisation of Islam in Britain, London: Social Affairs Unit, 2006, p. 2

40. Olivier ESTEVES, op. cit., p. 145.

41. " Rushdie broke Islamic Pact », The Guardian, 17 December 1991.

42. Stella ETCHEPARE, «Ruptures et dissensions au sein de la communauté musulmane britannique: quand les musulmans mettent en garde contre les dérives intégristes ", Lucienne Germain \& Didier Lassalle (dir.), Les relations interethniques dans l'aire anglophone: entre collaboration (s) et rejet(s), Paris : L'Harmattan, 2009, p. 69.

43. John SOLOMOS, Race and Racism in Britain (2 ${ }^{\text {nd }}$ edition), London: Macmillan, 1993, p. 223.

44. D'autres associations, radicales mais «participationnistes" (c'est-à-dire ne rejetant pas le dialogue avec les pouvoirs publics) ont été consultées à plusieurs reprises : Islamic Human Rights Commission, Muslim Association of Britain, Mawdudist Network; Muslim Public Affairs Committee etc.

45. SINGH \& SINGH TATLA, p. 138.

46. SINGH \& SINGH TATLA, p. 140. (traduction de l'auteur)

47. "Theatre community defends 'courageous' Birmingham Rep », The Guardian, Tuesday 21 December 2004, [http://www.guardian.co.uk/uk/2004/dec/21/arts.religion] 
48. SINGH \& SINGH TATLA, p. 140.

49. Pour plus de détails, voir Vincent LATOUR, «La communauté musulmane : une émergence tardive mais une installation durable dans le paysage politique et institutionnel britannique ", Hérodote $n^{\circ} 137$, revue de géopolitique, "Géopolitique des Iles britanniques ", sous la direction de Béatrice Giblin et Yves Lacoste. Paris : Edition La Découverte, juin 2010.

50. SINGH \& SINGH TATLA, p. 127.

51. «Enoch Powell's 'Rivers of Blood' speech », The Daily Telegraph, 6 November 2007. [http:// www.telegraph.co.uk/comment/3643826/Enoch-Powells-Rivers-of-Blood-speech.html] (lien actif le 17 juin 2011).

52. POULTER, Ethnicity, Law and Human Rights, 293-297, cité dans SINGH \& SINGH TATLA, p. 129.

53. SINGH \& SINGH TATLA, p. 130.

54. La candidature de l'élève n'avait pas été rejetée parce qu'il était sikh mais parce qu'il portait un turban.

55. SINGH \& SINGH TATLA, p. 132.

56. All England Law Report, Vol. 3, London : Butterworth \& Co, 1983, 1062, cité dans SINGH \& SINGH TATLA, p. 132.

57. Justifiant ainsi l'emploi d'une majuscule pour le substantif.

58. "Any attempt to wear a safety helmet..... [which] would, by virtue of any statutory provision or rule of law, be imposed on a Sikh who is on a construction site shall not apply to him at any time when he is wearing a turban.», cité dans SINGH \& SINGH TATLA, p. 134.

59. SINGH \& SINGH TATLA, p. 135.

60. Rob BERKELEY (with research by Savita Vija), Right to Divide? Faith Schools and Community Cohesion, London: Runnymede Trust, December 2008. [http://www.runnymedetrust.org/ uploads/publications/Summaries/RightToDivide-Summary.pdf] (lien actif le 17 juin 2011)

61. Patrick WINTOUR, "Minister gives schools right to ban Muslim veil », The Guardian, Tuesday 20 March 2007. (17 juin 2011).

62. En 2003, Shabina Begum alors âgée de 13 ans, fut exclue d'un établissement de Luton, Denbigh High School, pour port du jilbab (qui n'occulte pas le visage mais dissimule l'ensemble de corps et la chevelure). L'élève gagna son procès contre l'école en première instance, avant de perdre en appel puis à la Chambre de Lords, qui, en mars 2006, jugea que son exclusion ne contrevenait pas au Human Rights Act de 1998. Avec prudence, les Lords dirent que ce jugement n'avait ni portée générale, vocation à se prononcer sur les prescriptions vestimentaires en vigueur chez les musulmans. [http://www.publications.parliament.uk/pa/ld200506/ldjudgmt/jd060322/ begum-1.htm] (17 juin 2011). Par ailleurs, en novembre 2006 Aishah Azmi fut licenciée pour port du niqab par l'autorité locale de Kirklees qui l'employait en qualité d'auxiliaire d'enseignement avant d'être déboutée à deux reprises par la justice. A l'occasion de l'affaire Azmi Vs Kirklees $\mathrm{MBC}$, le Premier ministre Tony Blair commenta, contrairement à la coutume, la décision de justice pour dire qu'il approuvait le licenciement de la jeune femme.

63. Towards Greater Understanding. Meeting the needs of Muslim pupils in state schools. Information and Guidance for Schools, London: MCB, 2007, p. 26. [http://www.mcb.org.uk/downloads/ Schoolinfoguidancev2.pdf] (17 juin 2011).

64. Pour plus de détails, voir Vincent LATOUR, Revue Hérodote $n^{\circ} 137$, juin 2010.

65. Petite épée faisant partie des cinq préceptes du sikhisme ( les cinq $\mathrm{k} »)$. Elle est l'attribut exclusif des amrit-dhari, parfois appelés «Sikhs baptisés » (baptised Sikhs).

66. Voir par exemple Judith KNEEN, « Why do so many young people carry knives? », The Guardian , Tuesday 13 June 2006. [http://www.guardian.co.uk/education/2006/jun/13/ learnlessonplans.secondaryschools] (lien accessible le 5 juin 2011).

67. Rosa PRINCE, «Metal detectors in schools to stop knife crime », Daily Telegraph, 21 January 2011. 
68. «Key Messages concerning the Kirpan (a ceremonial sword in Sikhism): Guidance for Schools", [www.miltonkeynes.gov.uk/.../Guidance_on_Wearing_the_Kirpan_for_schools _May_2010.doc] (lien accessible le 17 juin 2011).

69. À condition, toutefois, que la lame soit dissimulée sous les vêtements et que le kirpan ne puisse pas être arraché.

70. Les vingt millions de Sikhs y représentent environ $2 \%$ de la population totale (soit 20 millions environ). Leur statut légal de minorité au Pendjab, le berceau historique de cette religion est en revanche controversé, depuis que la Cour Suprême indienne a refusé de leur reconnaître ce statut en 2008.

\section{RÉSUMÉS}

Au cours de la décennie passée (émeutes interethniques dans le Nord et les Midlands, attentats du 11 septembre et du 7 juillet 2005), le débat politique, universitaire et médiatique sur le multiculturalisme en tant que mode d'incorporation s'est focalisé de manière quasi exclusive (voire, obsessionnelle) sur la place de l'Islam et des musulmans dans la société britannique. A cet égard, le discours prononcé à Munich par David Cameron le 5 février 2011, si abondamment commenté, illustre l'ampleur de cette fixation, mais également sa permanence depuis les attentats du 11 septembre, voire depuis l'affaire Rushdie. En effet, l'irruption de la «communauté musulmane " sur le devant de la scène politico-médiatique britannique à la fin des années 1980 suite à la publication des Versets Sataniques tend à occulter la mobilisation coordonnée et constante des Sikhs du Royaume-Uni au cours des cinquante dernières années. Ils devancèrent largement les musulmans et les autres groupes minoritaires issus de l'immigration dans la formulation des demandes de reconnaissance culturelle.

Dix ans après les débuts de la remise en cause du « consensus multiculturel » né des émeutes des années 1980, il semble opportun de s'interroger sur deux stratégies distinctes de la visibilité, afin d'en identifier points de divergence et de convergence.

Cet article s'articulera autour de deux grandes parties. La première dressera un historique de leur présence au Royaume-Uni et s'intéressera aux prémices des visibilités musulmane et sikhe outre-manche, notamment par le biais des aspects cultuels. La deuxième tentera de mettre en parallèle les modalités de structuration communautaire et de demande de reconnaissance publique de ces deux groupes, y compris dans un contexte de profonde remise en cause apparente du multiculturalisme.

Over the past decade (interethnic riots in the North and the Midlands, 9/11 et 7/7/2005), the political academic and media debate on multiculturalism as a mode of incorporation has focused almost exclusively (or indeed, obsessively) on the place of Islam and Muslims in British society. David Cameron's Munich speech (5 February 2011), which has been so abundantly commented upon, illustrated the extent of that focalisation, as well as its permanence since $9 / 11$ or indeed, since the Rushdie Affair. As a matter of fact, the sudden emergence of the 'Muslim community' to the fore of the British political and media scene at the end of the 1980s following the publication of Rushdie's Satanic Verses has tended to overshadow the Sikhs' remarkably consistent and coordinated mobilisation over the past fifty years. Indeed, they preceded Muslims and other minority groups in the formulation of cultural demands.

A decade after the beginning of the questioning of the 'multicultural consensus', it seems both 
relevant and timely to look into two distinct visibility strategies, in order to identify both divergences and convergences. This paper will be divided into two parts. First, a historical record of the presence of Sikhs and Muslims in Britain shall be drawn, with a special reference to worship-related aspects, which provided early signs of both communities' visibility across the Channel. Then, part two shall endeavour to compare the Sikhs' and Muslims' modes of community mobilisation and the way each group has managed to formulate cultural demands, including in a context characterised, seemingly, by a profound questioning of multiculturalism.

\section{AUTEUR}

\section{VINCENT LATOUR}

Vincent Latour est Maître de conférences en civilisation britannique à l'Université Toulouse 2 Le Mirail et membre de l'équipe EEE (Europe, Européanité, Européanisation, FRE 3392/CNRS). Ses travaux s'inscrivent dans une démarche comparatiste et portent notamment sur la gestion de la diversité en Grande-Bretagne et en Europe. Parmi ses dernières publications, on notera sa contribution à l'ouvrage collectif Les politiques de la diversité. Expériences anglaise et américaine, («Bristol : un multiculturalisme moribond»), sous la direction de Denis Lacorne, Emmanuelle Le Texier \& Olivier Esteves, Paris : Presses Universitaires de Science Po, avril 2010, ou à la Revue Hérodote ( Conditions de l'émergence et de l'installation durable de la communauté musulmane dans le paysage politique et institutionnel britannique »), Hérodote $n^{\circ} 137$, « Géopolitique des Iles britanniques ", sous la direction de Béatrice Giblin et Yves Lacoste. Paris : Edition La Découverte, juin 2010. Il dirige par ailleurs, depuis 2010, le séminaire de l'équipe Europe, Européanité, Européanisation sur les signes religieux dans l'espace public en Europe. 